\title{
Molecular Predictors of Effective Implantation and Live Birth in IVF Programs
}

\author{
Elena G. Chukhnina ${ }^{1 *}$, PGS; Evgeny L. Kazachkov ${ }^{1}, \mathrm{PhD}, \mathrm{ScD}$; \\ Ekaterina E. Voropaeva ${ }^{1}, \mathrm{PhD}, \mathrm{ScD}$; Ella A. Kazachkova ${ }^{1}, \mathrm{PhD}, \mathrm{ScD}$; \\ Miroslava L. Polina ${ }^{2}, \mathrm{PhD}$; Natalya I. Douglas ${ }^{3}, \mathrm{PhD}, \mathrm{ScD}$ \\ ${ }^{1}$ South Ural State Medical University, Chelyabinsk, Russia \\ ${ }^{2}$ Women's Health Medical Center, Moscow, Russia \\ ${ }^{3}$ Medical Institute of North-Eastern Federal University named after M.K. Ammosov, Yakutsk, Russia
}

\begin{abstract}
The aim of the study was to improve the possibilities of predicting blastocyst implantation and live birth of ART programs in women of late reproductive age with tubal-peritoneal infertility based on immunohistochemical markers of the endometrium

Methods and Results: The results of IVF and IVF/ICSI programs were analyzed in 68 patients of late reproductive age (36-44 years of age) with tubal-peritoneal factor of infertility. Morphological examination of the endometrium was performed on Day 7 after confirmed ovulation in the cycle preceding ART. The expression of vitamin D receptors (VDR) and HOXA11 in endometrial stromal cells was assessed by immunohistochemical method. The effectiveness of using the endometrial markers VDR and HOXA11 as potential predictors of ART programs efficiency was confirmed by prognostic models. The levels of the stromal expression of VDR $<8.7 \%$ and HOXA $11<6.1 \%$ (probability $>0.27$ ) were determined to be favorable for successful blastocyst implantation. The expression levels of $\mathrm{VDR}<8.3 \%$ and HOXA $11<6.1 \%$ in endometrial stromal cells are prognostically favorable for live birth (probability $>0.19$ ) in women of late reproductive age with tubal-peritoneal infertility who undergoing ART treatment with their own oocytes.(International Journal of Biomedicine. 2021;11(4):532-537.)
\end{abstract}

Key Words: assisted reproductive technology $\bullet$ vitamin D receptor $\bullet$ HOXA11 $\bullet$ implantation $\bullet$ live birth

For citation: Chukhnina EG, Kazachkov EL, Voropaeva EE, Kazachkova EA, Polina ML, Douglas NI. Molecular Predictors of Effective Implantation and Live Birth in IVF Programs. International Journal of Biomedicine. 2021;11(4):532-537. doi:10.21103/ Article11(4)_OA21

\section{Abbreviations}

ART, assisted reproductive technology; AUC, the area under the ROC curve; IVF, in vitro fertilization; IHCM, immunohistochemical markers; ICSI, intracytoplasmic sperm injection; PCC, percentage correctly classified; RIF, recurrent implantation failure; VDR, vitamin D receptor.

\section{Introduction}

A new tendency in reproductive medicine is to improve the percentage of healthy perinatal outcomes for women using assisted reproductive technology (ART). ${ }^{(1)}$ Recurrent

*Corresponding author: Elena G. Chukhnina, PGS Department of Pathological Anatomy and Forensic Medicine, South Ural State Medical University, Chelyabinsk, Russia. E-mail: chuhninaeg@yandex.ru pregnancy losses, corresponding to the loss of three or more consecutive pregnancies up to 20 weeks, are noted in $1 \%-2 \%$ of couples, and their causes remain unexplained in more than half of the cases. ${ }^{(2)}$ The use of ART has improved the rates of pregnancy and live births. ${ }^{(3)}$ However, recurrent implantation failure (RIF), determined by the absence of fertilization after several IVF attempts, showed the need to study the effect of the endometrial pattern on the outcomes of ART programs. ${ }^{(4-7)}$ It is reported that suboptimal endometrial susceptibility and altered embryo-endometrial dialogue are responsible for 
two-thirds of implantation failures. ${ }^{(8)}$ An unknown reason for the functional inferiority of the endometrium in women with tubal-peritoneal infertility indicates the need to choose the most informative methods to predict the outcomes of ART programs. The female reproductive system is unique in relation to other tissues and systems due to predominantly postnatal differentiation and transformations during the menstrual cycle and pregnancy.

Homeobox genes (as known as $\mathrm{HOX}$ genes) are leading candidates for the regulation of endometrium differentiation in preparation for embryonic implantation. HOX genes encode proteins that act as transcription factors. ${ }^{(9,10)}$ Hoxa11/ HOXA11 expression is preserved in the adult endometrium of mice ${ }^{(11,12)}$ and humans. ${ }^{(12)}$ Some of the Hox/HOX genes participate in remodeling of the genitourinary tract, and regulation of endometrial proliferation and cell differentiation, with the formation of susceptibility of the uterine mucosa to implantation or apoptosis and tissue rejection during each menstrual cycle. ${ }^{(13-16)}$

Homeobox-deficient mice (Hoxa11) were found to be infertile due to stromal-specific endometrial defects that exclude decidual transformations. ${ }^{(17,18)}$

The prognostic potential of vitamin $\mathrm{D}$ to ensure female fertility is determined by its extensive receptor network in the organs of the reproductive system, including the endometrium. (19) Recent studies have confirmed the steroidogenic effect of the active form $1,25(\mathrm{OH}) 2 \mathrm{D}$ and participation in the modulation of cell proliferation, differentiation and apoptosis of cells of the reproductive organs. ${ }^{(20,21)}$ The effect of vitamin D on the endometrium is carried out by the formation in the cell nucleus of a ligand-independent transcription factor for the regulation of target genes and communication with cytosolic and membrane receptors. ${ }^{(22,23)}$

VDR is a part of a group of transcriptional regulators providing a variety of biological effects of $1,25(\mathrm{OH}) 2 \mathrm{D}$ and related compounds. ${ }^{(24)}$ The recognition of molecular and cellular endometrial breakdowns during the "implantation window" as one of the significant reasons for ineffective IVF attempts explains the interest in studying the mechanisms of local activity of VDR and $\mathrm{Hox} / \mathrm{HOX}$ genes.

The aim of the study was to improve the possibilities of predicting blastocyst implantation and live birth of ART programs in women of late reproductive age with tubalperitoneal infertility based on ICHM of the endometrium.

\section{Materials and Methods}

Our prospective cohort study included 68 women of late reproductive age (36-44 years of age) with tubal-peritoneal infertility who underwent ART programs at the Center of Obstetrics and Gynecology.

Inclusion criteria for the study were tubal factor of infertility, normal or reduced ovarian reserve with a preserved regular ovulatory menstrual cycle, normozoospermia or minor pathozoospermia of the husband (donor), use of the patient's own oocytes, embryos of good and excellent quality.

Exclusion criteria were infertility due to the absence of ovulation; endometriosis, uterine fibroids $4 \mathrm{~cm}$ or more, uterine factor of infertility, chronic active endometritis, HIV infection, hepatitis B and C, severe pathozoospermia, systemic diseases; somatic diseases in the stage of exacerbation or decompensation.

Depending on the outcomes of ART programs, 4 groups were formed, two of them according to the incidence of clinical pregnancy: Group $1(\mathrm{n}=18)$ - with pregnancy, Group $2(n=50)$ - with a negative result; and two groups according to the indicator of live birth (frequency of deliveries with a live fetus(es) [take-home baby]): Group $3(n=14)$ - with a favorable result, Group $4(n=54)$-with a negative result.

Endometrial sampling by aspiration biopsy using a Pipelle catheter during the period of the proposed "implantation window" was performed in 68 women in the cycle preceding the ART programs (on Days 17-25 of the menstrual cycle, depending on the results of ultrasound monitoring and on Day 7 after the peak of luteinizing hormone.

For histological examination, the standard method of fixation in $10 \%$ neutral formalin was used, followed by dehydration, degreasing and embedding in paraffin in a histological machine, according to the generally accepted technique. From paraffin blocks, sections with a thickness of $5 \mu \mathrm{m}$ were prepared, several (5-10) on 10-15 glass slides. After dewaxing, the sections were stained with hematoxylin and eosin; the avidin-biotin immunoperoxidase method was used for the immunohistochemical study of endometrial biopsies.

The polyclonal antibodies (PCAB) used in the study were designed to work with paraffin sections. Treatment options for dewaxed sections were selected depending on the manufacturer's instructions (Table 1). A Histophine detection system (Nichirei Corp., Japan) was used to visualize primary antibodies.

Table 1.

Panel of antibodies for immunohistochemical study.

\begin{tabular}{|c|c|c|c|}
\hline Antibodies & Clone & Working dilution & Manufacturer \\
\hline VDR & polyclonal & $1: 100$ & GeneTex, USA \\
\hline HOXA11 & polyclonal & $1: 250$ & GeneTex, USA \\
\hline
\end{tabular}

The stained samples were automatically classified by the program into 10 color channels, depending on the color and the intensity of staining. After classification, pseudo-color masks were applied to the structures of interest. In this case, the relative density ( $\%)$ of the studied structures was calculated in relation to the total area of the studied frame.

The results of VDR and HOXA11 expression were calculated by counting and finding the percentage of the relative density of stained endometrial stromal cells. Morphofunctional assessment of the endometrium was performed using licensed software (Morphology 5.2., Russia). The preparations were examined and photographed using a Primo Star microscope(Carl Zeiss, Germany), with the help of a Pixera Pro 150ES digital camera (Pixera, Japan) at an operating magnification of $\mathrm{x} 400$.

Embryo transfer was carried out in "fresh" cycles and cycles with the transfer of thawed embryos. For the pregnancy rate indicator, only clinical pregnancy (sonographic presence 
of fetal egg/eggs) was taken into account, and for live births - the number of ART programs that ended with live births/ fetuses.

All patients were informed about the purpose and design of the work and gave their consent to participate in the study and publish its results in the open press. The study was approved by the SUSMU Ethics Committee.

Statistical analysis was performed using the IBM SPSS Statistics for Windows, Version 22.0. Armonk, NY: IBM Corp.). The normality of distribution of continuous variables was tested by the Kolmogorov-Smirnov test with the Lilliefors correction and Shapiro-Wilk test. Continuous variables with normal distribution were presented as mean (standard deviation [SD]); non-normal variables were reported as median $(\mathrm{Me})$ and interquartile range (Q1-Q3). Means of 2 continuous normally distributed variables were compared by independent samples Student's t test. Differences of continuous variables departing from the normal distribution, even after transformation, were tested by the MannWhitney U-test. Threshold values (cut-off point) were calculated by the ROC analysis method. To construct a prognostic model and estimate OR (odds ratio), we used the method of multiple logistic regression; 95\% CI (confidence interval) was calculated. A value of $P<0.05$ was considered significant.

\section{Results}

The onset of clinical pregnancy was detected in $18(26.5 \%)$ patients with their own oocytes in ART programs. In Group 1 and Group 2, VDR expression in the endometrial stroma was $7.35(4.9 ; 8.1) \%$ and $8.56 .3 ; 9.8) \%(P=0.016)$, respectively, and HOXA11 expression - 5.1(4.3;6.1)\% and 7.4(5.4; 8.7)\% ( $P=0.001)$, respectively.

The AUC was determined for implantation markers: VDR of the stroma $-0.739 \pm 0.063(P=0.003)$ and HOXA11 $0.767 \pm 0.064(P=0.000)$.

The threshold value of VDR expression in endometrial stromal cells at the cut-off point was $8.7 \%$. The sensitivity and specificity of the method were $100 \%$ and $40 \%$, respectively (Fig.1). The threshold value of HOXA11 expression in endometrial stromal cells, calculated for successful implantation at the cut-off point, was $6.1 \%$. The sensitivity and specificity of the method were $80 \%$ and $73 \%$, respectively (Figure 2).

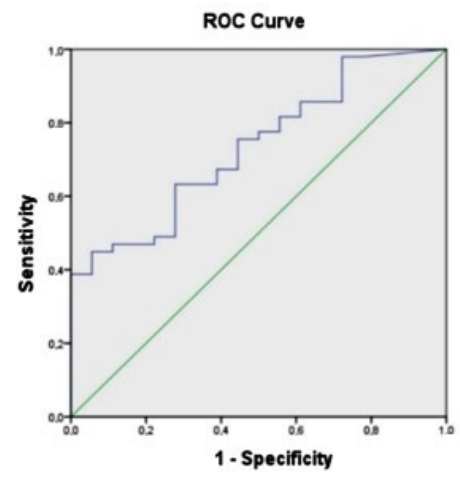

Fig.1. ROC-curve of the relationship between successful implantation (the onset of clinical pregnancy) and the VDR expression in endometrial stromal cells

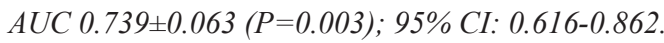

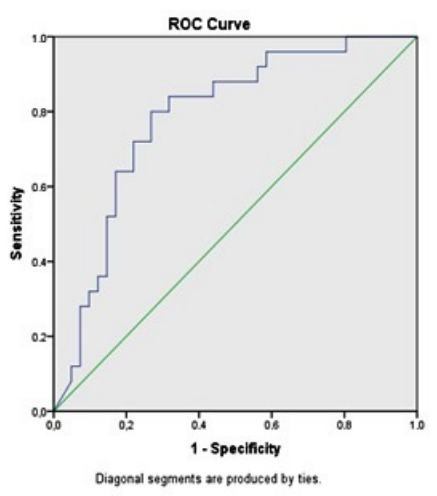

Fig.2. ROC-curve of the relationship between successful implantation (the onset of clinical pregnancy) and the HOXA11 expression in endometrial stromal cells.

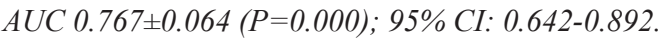

To create a mathematical model that allows predicting implantation, we used multiple regression analysis with forced inclusion of two variables: VDR, HOXA11 (Table 2).

Table 2.

Calculation of the blastocyst implantation prognosis

\begin{tabular}{|c|c|c|c|c|}
\hline Parameter & В & Value & OR(Exp(в) & $95 \%$ CI for OR \\
\hline HOXA11 & -0.487 & 0.003 & 0.615 & {$[0.444 ; 0.850]$} \\
\hline VDR & -0.302 & 0.012 & 0.740 & {$[0.585 ; 0.935]$} \\
\hline Constant & 4.121 & 0.005 & 61.649 & \\
\hline
\end{tabular}

The equation was as follows:

$$
p=\frac{1}{1+e^{-\left(4.121-0.487 \cdot x_{1}-0.302 \cdot x_{2}\right)}},
$$

where $x_{1}$ is the value of HOXA11 expression in the endometrial stroma, $x$ is the value of VDR expression in endometrial stromal cells, $p$ is the probability of implantation.

If the probability calculated by the model is $>0.27$, then the onset of implantation is predicted. The resulting model is statistically significant $(P<0.001)$. Model sensitivity $(83.3 \%)$, specificity $(74.0 \%)$, and PCC $(76.5 \%)$ indicate a high predictive ability.

The ROC curves for predicting blastocyst implantation, taking into account the expression of individual markers in the endometrium, are presented in Figure 3.

Live birth in ART programs occurred in 14(21.7\%) women. The expression of IHCM in the endometrial stroma in Groups 1 and 2 was as follows: $7.1(4.9 ; 8.0) \%$ and $8.5(6.1 ; 9.8) \%$, respectively, for $\mathrm{VDR}(\mathrm{P}=0.240) ; 5.0(4.3 ; 6.1) \%$ and $7.1(5.3 ; 8.7) \%$, respectively, for HOXA11 $(P=0.006)$. In patients of Group 1, the decrease in HOXA11 expression turned out to be statistically significant $(P=0.006)$.

The AUC of IHCM expression in women with their own oocytes was $0.728 \pm 0.067(P=0.009)$ for the VDR in the stroma and $0.744 \pm 0.071(P=0.005)$ for HOXA11.

The value of the threshold parameter of HOXA11 expression, prognostically favorable for live birth, was 
$6.1 \%$. The sensitivity and specificity of the method were $80 \%$ and $63 \%$, respectively. The threshold value of VDR expression in stromal cells was calculated and turned out to be prognostically favorable for live birth was $8.3 \%$. The sensitivity and specificity of the method were $93 \%$ and $53 \%$, respectively.

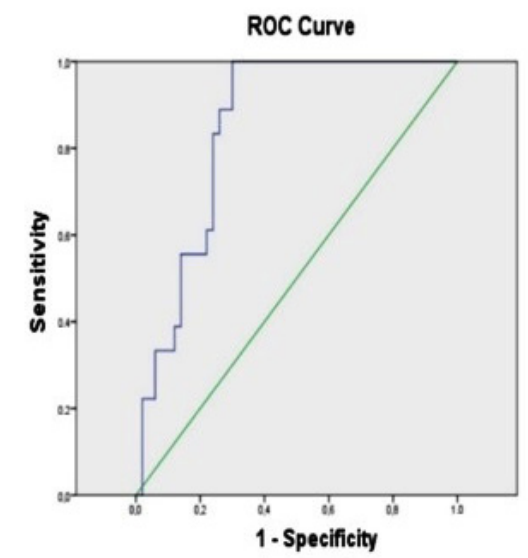

Fig.3. AUC for the blastocyst implantation prognosis model.

$A U C=0.846(P=0.000) ; 95 \% C I: 0.756-0.935$.

To create a mathematical model that allows predicting live birth, we used multiple regression analysis with the forced inclusion of two variables: VDR, HOXA11 (Table 3).

Table 3

Calculation of the live birth prognosis

\begin{tabular}{|c|c|c|c|c|}
\hline Parameter & B & Value & OR(Exp(в) & $95 \%$ CI for OR \\
\hline HOXA11 & -0.308 & 0.030 & 0.735 & {$[0.556 ; 0.971]$} \\
\hline VDR & -0.329 & 0.006 & 0.720 & {$[0.568 ; 0.912]$} \\
\hline Constant & 2.825 & 0.033 & 16.860 & \\
\hline
\end{tabular}

The equation was as follows:

$$
P=\frac{1}{1+e^{-\left(2.825-0.308 \cdot x_{1}-0.329 \cdot x_{2}\right)}},
$$

where $x_{1}$ is the value of HOXA11 expression in the endometrial stroma, $x_{2}$ is the value of VDR expression in endometrial stromal cells, $p$ is the probability of live birth.

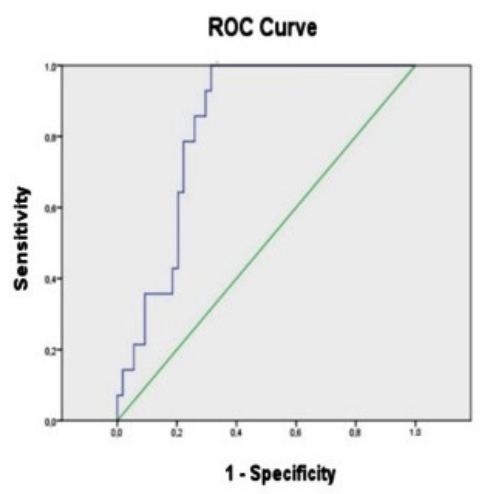

Fig. 4. AUC for the live birth prediction model $A U C=0.831$ ( $P=0.000)$; 95\% CI: 0.736-0.925.
The probability calculated by the model of $>0.19$ means a favorable prognosis for live birth $(P<0.001)$. Model sensitivity $(86.0 \%)$, specificity $(70.0 \%)$, and PCC $(78.6 \%)$ indicate a high predictive ability. ROC curves for predicting live birth, taking into account the expression of individual markers in the endometrium, are shown in Figure 4.

\section{Discussion}

Predicting the outcomes of ART programs is possible if the "subtle" endometrial patterns of fertility regulation are taken into account. The results obtained showed that effective interaction between the endometrium and the blastocyst during the "implantation window" suggests a lower level of VDR expression in endometrial stromal cells.

These results explain the data of studies indicating a decrease in VDR expression in the middle stage of the secretion phase (during the "implantation window") in healthy women and a decrease in the antiproliferative effect at low levels of VDR expression. ${ }^{(25)}$ This is probably due to the progesteronelike activity of vitamin $\mathrm{D}$ along with the limitation of local proinflammatory reactions necessary for blastocyst implantation. The susceptibility of the endometrium during the implantation period was due to a decrease in VDR activity, which probably affects not only the metabolic profile of cell subpopulations, but also the ratio of immune cells.

The data we obtained differ from the results of researchers who indicated the relationship between positive outcomes of IVF and IVF/ICSI programs and increased VDR expression. In the study by J. Guo et al., ${ }^{(26)}$ for example, the absence of significant statistical differences $(P=0.083)$, the younger age of women with pregnancy $(P=0.032)$, the small sample size $(n=16)$, and the study of endometrial samples at different phases of the menstrual cycle in different women are factors that limit the significance of the results.

In earlier studies, we identified a decrease in the expression of the VDR protein in the stromal epithelium ( $P=0.016)$ of women with pregnancy, as opposed to the glandular layer. ${ }^{(27)}$ It has been calculated that a $1 \%$ decrease in VDR expression in the stroma increases the chance of a favorable outcome by 1.35 times. $\left({ }^{28)}\right.$

The combination of the optimal expression level of VDR and HOXA11 has a beneficial effect on implantation and the outcomes of IVF and IVF/ICSI programs. Therefore, it is possible to assert that a lower expression of endometrial markers is crucial for the synchronized dialogue between the endometrium and the embryo, as well as for live birth.

Our research results differ from those given in the literature. Thus, Makker A. et al. ${ }^{(29)}$ revealed no differences in protein expression in patients with infertility and uterine fibroids that do not deform the cavity, compared with healthy fertile women. According to other data, ${ }^{(30)}$ the minimum level of HOXA11 in the endometrium was found only in infertility of unknown origin, which was significantly lower than in the control group, in contrast to women with other types of infertility $(P=0.005)$. A decrease in HOXA11 expression in the middle of the secretory phase during implantation and in the early stages of pregnancy was determined only in the 
glandular endometrium, in contrast to stromal cells, in which high expression persisted throughout the menstrual cycle. ${ }^{(31)}$

\section{Conclusion}

The effectiveness of using the endometrial markers VDR and HOXA11 as potential predictors of ART programs efficiency is confirmed by prognostic models. The levels of the stromal expression of $\mathrm{VDR}<8.7 \%$ and $\mathrm{HOXA} 11<6.1 \%$ (probability $>0.27$ ) were determined to be favorable for successful blastocyst implantation. The expression levels of VDR $<8.3 \%$ and HOXA $11<6.1 \%$ in endometrial stromal cells are prognostically favorable for live birth (probability $>0.19$ ) in women of late reproductive age with tubal-peritoneal infertility who undergoing ART treatment with their own oocytes.

\section{Competing Interests} interests.

The authors declare that they have no competing

\section{Sources of Funding}

This research was funded by RFBR and Chelyabinsk Region, Project number 20-415-740014.

\section{References}

1. Kushnir VA, Barad DH, Albertini DF, Darmon SK, Gleicher N. Systematic review of worldwide trends in assisted reproductive technology 2004-2013. Reprod Biol Endocrinol. 2017 Jan 10;15(1):6. doi: 10.1186/s12958-016-0225-2.

2. Ford HB, Schust DJ. Recurrent pregnancy loss: etiology, diagnosis, and therapy. Rev Obstet Gynecol. 2009 Spring;2(2):76-83.

3. Farquhar C, Marjoribanks J. Assisted reproductive technology: an overview of Cochrane Reviews. Cochrane Database Syst Rev. 2018 Aug 17;8(8):CD010537. doi: 10.1002/14651858.CD010537.pub5.

4. Simon A, Laufer N. Repeated implantation failure: clinical approach. Fertil Steril. 2012 May;97(5):1039-43. doi: 10.1016/j.fertnstert.2012.03.010.

5. Matsumoto L, Hirota Y, Saito-Fujita T, Takeda N, Tanaka T, Hiraoka T, Akaeda S, Fujita H, Shimizu-Hirota R, Igaue S, Matsuo M, Haraguchi H, Saito-Kanatani M, Fujii T, Osuga Y. HIF $2 \alpha$ in the uterine stroma permits embryo invasion and luminal epithelium detachment. J Clin Invest. $2018 \mathrm{Jul}$ 2;128(7):3186-3197. doi: 10.1172/JCI98931.

6. Herington JL, Guo Y, Reese J, Paria BC. Gene profiling the window of implantation: Microarray analyses from human and rodent models. J Reprod Health Med. 2016 Dec;2(Suppl 2):S19-S25. doi: 10.1016/j.jrhm.2016.11.006.

7. Lv H, Li X, Du J, Ling X, Diao F, Lu Q, Tao S, Huang L, Chen S, Han X, Zhou K, Xu B, Liu X, Ma H, Xia Y, Shen H, Hu Z, Jin G, Guan Y, Wang X. Effect of endometrial thickness and embryo quality on live-birth rate of fresh IVF/ICSI cycles: a retrospective cohort study. Reprod Biol Endocrinol. 2020 Aug 21;18(1):89. doi: 10.1186/s12958-020-00636-6.
8. Tomari H, Kawamura T, Asanoma K, Egashira K, Kawamura K, Honjo K, Nagata Y, Kato K. Contribution of senescence in human endometrial stromal cells during proliferative phase to embryo receptivity $\dagger$. Biol Reprod. 2020 Jun 23;103(1):104-113. doi: 10.1093/biolre/ioaa044.

9. Du H, Taylor HS. The Role of Hox Genes in Female Reproductive Tract Development, Adult Function, and Fertility. Cold Spring Harb Perspect Med. 2015 Nov 9;6(1):a023002. doi: 10.1101/cshperspect.a023002.

10. Xu B, Geerts D, Qian K, Zhang H, Zhu G. Myeloid ecotropic viral integration site 1 (MEIS) 1 involvement in embryonic implantation. Hum Reprod. 2008 Jun;23(6):1394406. doi: 10.1093/humrep/den082.

11. Hsieh-Li HM, Witte DP, Weinstein M, Branford W, Li H, Small K, Potter SS. Hoxa 11 structure, extensive antisense transcription, and function in male and female fertility. Development. 1995 May;121(5):1373-85.

12. Taylor HS, Vanden Heuvel GB, Igarashi P. A conserved Hox axis in the mouse and human female reproductive system: late establishment and persistent adult expression of the Hoxa cluster genes. Biol Reprod. 1997 Dec;57(6):1338-45. doi: 10.1095/biolreprod57.6.1338.

13. Taylor HS, Arici A, Olive D, Igarashi P. HOXA10 is expressed in response to sex steroids at the time of implantation in the human endometrium. J Clin Invest. 1998 Apr 1;101(7):1379-84. doi: 10.1172/JCI1057.

14. Magnusson M, Brun AC, Miyake N, Larsson J, Ehinger M, Bjornsson JM, Wutz A, Sigvardsson M, Karlsson S. HOXA10 is a critical regulator for hematopoietic stem cells and erythroid/megakaryocyte development. Blood. 2007 May 1;109(9):3687-96. doi: 10.1182/blood-2006-10-054676.

15. Gellersen B, Brosens JJ. Cyclic decidualization of the human endometrium in reproductive health and failure. Endocr Rev. 2014 Dec;35(6):851-905. doi: 10.1210/er.20141045.

16. Lu Z, Hardt J, Kim JJ. Global analysis of genes regulated by HOXA10 in decidualization reveals a role in cell proliferation. Mol Hum Reprod. 2008 Jun;14(6):357-66. doi: 10.1093/molehr/gan023.

17. Taylor HS, Igarashi P, Olive DL, Arici A. Sex steroids mediate HOXA11 expression in the human peri-implantation endometrium. J Clin Endocrinol Metab. 1999 Mar;84(3):112935. doi: $10.1210 /$ jcem.84.3.5573.

18. Gendron RL, Paradis H, Hsieh-Li HM, Lee DW, Potter SS, Markoff E. Abnormal uterine stromal and glandular function associated with maternal reproductive defects in Hoxa-11 null mice. Biol Reprod. 1997 May;56(5):1097-105. doi: 10.1095/ biolreprod56.5.1097.

19. Adams JS, Hewison M. Unexpected actions of vitamin D: new perspectives on the regulation of innate and adaptive immunity. Nat Clin Pract Endocrinol Metab. 2008 Feb;4(2):8090. doi: $10.1038 /$ ncpendmet 0716 .

20. Voulgaris N, Papanastasiou L, Piaditis G, Angelousi A, Kaltsas G, Mastorakos G, Kassi E. Vitamin D and aspects of female fertility. Hormones (Athens). 2017 Jan;16(1):5-21. doi: 10.14310/horm.2002.1715.

21. Monastra G, De Grazia S, De Luca L, Vittorio S, Unfer V. Vitamin D: a steroid hormone with progesterone-like activity. Eur Rev Med Pharmacol Sci. 2018 Apr;22(8):2502-2512. doi: 10.26355/eurrev_201804_14845.

22. Lerchbaum E, Obermayer-Pietsch B. Vitamin D and 
fertility: a systematic review. Eur J Endocrinol. 2012 May;166(5):765-78. doi: 10.1530/EJE-11-0984.

23. Cermisoni GC, Alteri A, Corti L, Rabellotti E, Papaleo E, Viganò P, Sanchez AM. Vitamin D and Endometrium: A Systematic Review of a Neglected Area of Research. Int J Mol Sci. 2018 Aug 8;19(8):2320. doi: 10.3390/ijms19082320.

24. Katayama Y. Vitamin D receptor: A critical regulator of inter-organ communication between skeletal and hematopoietic systems. J Steroid Biochem Mol Biol. 2019 Jun;190:281-283. doi: 10.1016/j.jsbmb.2019.02.001.

25. Zelenko Z, Aghajanova L, Irwin JC, Giudice LC. Nuclear receptor, coregulator signaling, and chromatin remodeling pathways suggest involvement of the epigenome in the steroid hormone response of endometrium and abnormalities in endometriosis. Reprod Sci. 2012 Feb;19(2):152-62. doi: 10.1177/1933719111415546.

26. Guo J, Liu S, Wang P, Ren H, Li Y. Characterization of VDR and CYP27B1 expression in the endometrium during the menstrual cycle before embryo transfer: implications for endometrial receptivity. Reprod Biol Endocrinol. 2020 Mar 17;18(1):24. doi: 10.1186/s12958-020-00579-y.

27. Chukhnina EG, Kazachkov EL, Voropaeva EE, Kazachkova EA, Polina ML, Douglas NI. The Effect of Vitamin D Metabolic
Status and Endometrial Immune Patterns on the Outcomes of ART Programs. International Journal of Biomedicine, 2021;11(2):188-196. doi:10.21103/Article11(2) OA11.

28. Chukhnina EG, Voropaeva EE, Kazachkov EL, Kazachkova EA. Influence of vitamin D receptors expression on clinical outcomes of assisted reproductive technology programs. Ural Medical Journal. 2020;6(189):63-68. doi:10.25694/URMJ.2020.06.15

29. Makker A, Goel MM, Nigam D, Bhatia V, Mahdi AA, Das V, Pandey A. Endometrial Expression of Homeobox Genes and Cell Adhesion Molecules in Infertile Women With Intramural Fibroids During Window of Implantation. Reprod Sci. 2017 Mar;24(3):435-444. doi: 10.1177/1933719116657196.

30. C. Margioula-Siarkou C, Petousis S, Milias S, Ravanos K, Kalogiannidis I, Mavromatidis G et al. Endometrial expression of Leukemia Inhibitory Factor (LIF), LIF-receptor and HOXA11 but not HOXA-10 is significantly impaired in women with unexplained infertility during implantation window. Eur J Obstet Gynecol Reprod Biol. 2016;206:E165-E1666. doi: 10.1016/j.ejogrb.2016.07.410

31. Wang LF, Luo HZ, Zhu ZM, Wang JD. Expression of HOXA11 gene in human endometrium. Am J Obstet Gynecol. 2004 Sep;191(3):767-72. doi: 10.1016/j.ajog.2004.02.069. 